\title{
Síndrome metabólica: bases moleculares e fundamentos da interação com obesidade
}

\section{Metabolic syndrome: molecular basis and reasons for interaction with obesity}

Ana Paula Moreira Bezerral

Diana Magalhães de Oliveira²

' Nutricionista, Curso de Mestrado Acadêmico em Nutrição e Saúde, Universidade Estadual do Ceará. Fortaleza, CE, Brasil.

2 Professora Adjunto da Universidade Estadual do Ceará. Programa de Pós-Graduaçãa em Biotecnologia da Rede Nordeste de Biotecnologia e Curso de Mestrado Acadêmico em Nutrição e Saúde. Fortaleza, CE, Brasil.

Correspondência / Correspondence Diana Magalhães de Oliveira E-mail:diana.magalhaes@uece.br

\section{Resumo}

Quando Gerald Reaven, no final da década de 1980, introduziu o conceito de síndrome metabólica (SM), esta se tornou tópico de intensa discussão científica. A predisposição genética, a alimentação inadequada e a inatividade física estão entre os principais fatores que contribuem para o surgimento da SM, cuja prevenção primária é um desafio mundial contemporâneo. Embora a patogênese da SM não esteja completamente elucidada, seus diferentes componentes estão possivelmente associados à resistência à insulina. Na SM, ocorre uma provável interação dos fatores genéticos, metabólicos e ambientais, incluindo a dieta. $\mathrm{O}$ papel dos fatores dietéticos na presença e no desenvolvimento da SM não está estabelecido de forma definitiva. Aspectos alimentares e nutricionais presentes na vida moderna, particularmente associados aos hábitos alimentares inadequados e ao sedentarismo, desempenham expressivo papel na gênese da obesidade, representando cadeia de causalidade de fácil observação, mas de difícil intervenção. Nesse sentido, esta revisão trata do estado da arte e das circunstâncias que caracterizam a SM, evidenciando o conhecimento dos eventos moleculares (genes e proteínas) que regulam a adipogênese e são importantes para o entendimento da obesidade como fator de risco da SM.

Palavras-chave: Síndrome X metabólico (SM). Obesidade. Genes reguladores. Adipogenia. 


\section{Abstract}

When Gerald Reaven, in late 1980's, introduced the term metabolic syndrome (MS), it soon became a theme of great scientific debate. Genetic background, onadequate diet and sedentary lifestile contribute to MS outcome, whose overall prevention is now believed to be a world wide challenge. Although MS pathogenesis is not completely clarified, its various components are thought to be associated to insulin resistence. Maybe in MS there is interation among genetic, metabolic and environmental features, including diet. Diet role on the development of MS is not yet fully established on a precise or definitive way. Food and nutrition aspects in modern life, especially associated to questionable feeding behaviors and / or sedentary lifestyle, play significant role in obesity, representing a causality chain that is easily observed but hard to intervene. So this article aims at reviewing the state-of-the-art and circumstances that characterize MS, highlighting the knowledge on molecular events (genes and proteins) that regulate adipogenesis and help understand obesity as risk factor for MS.

Key words: Metabolic Syndrome X (MS) Obesity, Genes, Regulator Adipogenesis.

\section{Introdução}

Esta revisão objetiva apresentar e discutir estudos que caracterizam a síndrome metabólica (SM) e sua relação com a obesidade. Trabalhos que abordam pontos de vista físico, clínico, bioquímico e genético dessa síndrome são analisados, evidenciando suas bases moleculares, que vêm ultimamente sendo desvendadas e dissecadas, a fim de melhor entender a adipogênese e a obesidade.

Em humanos adultos, o acúmulo de múltiplos fatores de risco cardiovascular já era observado desde o início do século XX., ${ }^{1,2}$ Mais recentemente, porém, acúmulos similares de certos fatores cardíacos receberam renovada atenção e termos como o quarteto mortal, ${ }^{3}$ a síndrome de resistência à insulina, ${ }^{4}$ a Síndrome Metabólica $(\mathrm{SM}),{ }^{5}$ síndrome $\mathbf{X},{ }^{6}$ ou ainda síndrome plurimetabólica, foram propostos com o intuito de descrever a conexão entre obesidade, resistência à insulina, hipertensão arterial sistêmica (HAS), dislipidemia, diabetes mellitus tipo 2 (DMT2), e doença cardiovascular aterosclerótica (DCA). Pode-se dizer, então, que a definição dessa síndrome varia em termos de quais indicadores se apresentam e dos pontos de corte usados. ${ }^{2}$ 
Há evidências recentes, porém controversas, ${ }^{7}$ com informações substanciais sobre obesidade infantil em associação com resistência à insulina, inflamação e outros fatores de risco e seu papel coletivo para o risco aumentado de DCA e DMT2. A constelação desses fatores de risco cardiovascular inter-relacionados em adultos veio a ser conhecida por síndrome metabólica (SM), uma denominação que se mostra útil tanto nos contextos clínicos quanto de pesquisa ${ }^{2}$ e que recebeu recente atualização consensual de diretrizes mundiais para seu estudo, diagnóstico e prevenção. ${ }^{8,9}$ A SM tem sido caracterizada, portanto, pela ocorrência de distúrbios complexos representados por um conjunto de fatores de risco cardiovascular, usualmente relacionados à deposição central de gordura e resistência à insulina. ${ }^{10}$

Outras condições associadas com a SM incluem inatividade física, envelhecimento, desequilíbrio hormonal e predisposição genética. Alguns indivíduos são geneticamente suscetíveis à resistência à insulina. No entanto, fatores como excesso de gordura corporal e inatividade física podem favorecer o desenvolvimento da SM. É muito comum a associação de resistência à insulina com obesidade abdominal, mas as bases moleculares dos mecanismos biológicos entre resistência à insulina e fatores de risco metabólico não estão ainda totalmente esclarecidos e parecem ser realmente bastante complexos. ${ }^{11}$ Chama a atenção, inclusive, o fato recém-levantado ${ }^{9}$ de que a estabilidade da SM, especialmente em adolescentes, é baixa, o que suscita questões sobre a utilidade do conceito de SM num contexto clínico.

De acordo com a Organização Mundial de Saúde (OMS), ${ }^{12,13}$ a SM é um distúrbio metabólico complexo que se caracteriza pela associação de tolerância à glicose prejudicada / diabetes mellitus e/ou resistência insulínica, além de dois ou mais dos seguintes fatores: HAS (valores superiores a 140/90mmHg); hipertrigliceridemia (concentrações plasmáticas maiores que $150 \mathrm{mg} / \mathrm{dl}$ ) e/ou concentrações séricas de lipoproteína de alta densidade (HDL-c) inferiores a 35mg/dl e 39mg/dl, em homens e mulheres, respectivamente; obesidade ou adiposidade central/abdominal (definida por relação cintura-quadril superior a 0,90 para o sexo masculino e 0,85 para o feminino) e/ou Índice de Massa Corporal (IMC $\mathrm{IM}^{\mathrm{a}}$ ) maior que $30 \mathrm{~kg} / \mathrm{m}^{2}$; microalbuminúria (taxa de excreção urinária de albumina maior ou igual a $20 \mu \mathrm{g} / \mathrm{min}$ ou razão albumina: creatinina maior ou igual a $30 \mathrm{mg} / \mathrm{g}$ ).

As associações e agrupamentos desses fatores são conhecidos há décadas, o que serviu para demonstrar claramente que a SM é comum, com alta prevalência mundial, e continua crescendo e ocorrendo nos grupos populacionais de sedentários e obesos. ${ }^{14}$ Resultado disso, a SM agora surge como grave problema de saúde pública, além de continuar um grave problema clínico.

A incidência de SM tem crescido assustadoramente nos últimos anos e estima-se que mais de 50 milhões de norte-americanos sejam acometidos por essa doença crônica não-transmissível

a $\quad$ IMC = divisão da massa corporal em quilogramas $(\mathrm{Kg})$ pela estatura em metros ao quadrado. 
(DCNT). ${ }^{11}$ Em 2001, o National Cholesterol Education Program $\left(\mathrm{NCEP}^{\mathrm{b}}\right)$, EUA, descreveu que o diagnóstico de SM é determinado pela presença de três ou mais das seguintes afecções: obesidade abdominal $^{\mathrm{c}}, \mathrm{HAS}^{\mathrm{d}}$, tolerância à glicose prejudicada ${ }^{\mathrm{e}}$, hipertrigliceridemia ${ }^{\mathrm{f}}$, baixas concentrações de HDL-c ${ }^{g}$. Ilustrativamente, a listagem abaixo representaria um repertório completo típico de SM.

- obesidade abdominal (excessivo acúmulo de gordura no abdômen);

- dislipidemia aterogênica (desordens de gordura sanguínea e colesterol - hipertrigliceridemia, baixo HDL e alto LDL - que provocam acúmulo de placas gordurosas na parede das artérias);

- elevada pressão arterial;

- resistência à insulina ou intolerância à glicose;

- estado pró-trombótico (elevados níveis de fibrinogênio ou inibidor do ativador de plasminogênio-1 na corrente sanguínea);

- estado pró-inflamatório (elevados níveis de proteína C-reativa na corrente sanguínea).

\section{Quadro representativo do repertório completo de condições possíveis, nem sempre todas coexistindo, na SM. Adaptado a partir de NCEP (2001)}

Segundo Lorenzo et al., ${ }^{15}$ a definição do NCEP detecta maior número de indivíduos em risco de diabetes $(48,7 \%)$ do que a definição da OMS (41,3\%), uma vez que a SM constitui fator preditivo de desenvolvimento de diabetes mellitus, independentemente de outros fatores de risco.

Apesar de ser relativamente bem estudada e aceita como importante problema de saúde, alguma controvérsia ainda persiste quanto a ser a SM uma verdadeira síndrome ${ }^{\mathrm{h}}$ ou não - isto é, se ela seria apenas uma mistura de fenótipos não-relacionados. ${ }^{14}$ Sabe-se que a SM não pode ser usada como indicador absoluto de risco, uma vez que ela não contém per se muitos dos fatores

b O NCEP, The National Cholesterol Education Program, criado em 1985 pelo National Heart, Lung, and Blood Institute (NHLBI) dos Estados Unidos, tem por objetivo a educação continuada de profissionais e do público em geral acerca dos benefícios da redução dos níveis de colesterol como maneira de reduzir os riscos das doenças coronarianas.

c Obesidade abdominal é determinada por valores de circunferência de cintura superiores a $102 \mathrm{~cm}$ e $88 \mathrm{~cm}$, em homens e mulheres, respectivamente.

d Hipertensão arterial é definida por níveis pressóricos iguais ou maiores que 130/85mmHg.

e Glicemia de jejum entre 110 e $125 \mathrm{mg} / \mathrm{dl}$.

f Hipertrigliceridemia considerada com valores iguais ou superiores a $150 \mathrm{mg} / \mathrm{dl}$.

g HDL-c: taxas inferiores a $40 \mathrm{mg} / \mathrm{dl}$ para homens e $50 \mathrm{mg} / \mathrm{dl}$ para mulheres.

h Uma síndrome é simplesmente um agrupamento de fatores que aparecem frequentemente mais juntos do que naturalmente ocorreriam ao acaso e cuja causa para isso pode ser tida como incerta ou multifatorial. A SM atende esse critério para inclusão como síndrome. 
que determinam o risco absoluto - por exemplo, idade, sexo, fumo e níveis de LDL. Ainda assim, os indivíduos com SM apresentam duas vezes mais riscos de desenvolver doença cardiovascular nos próximos cinco a dez anos, e cinco vezes mais chances de desenvolver DMT2. Além disso, os indivíduos com a tríade (obesidade, DMT2 e inflamação) geralmente apresentam um estado pró-trombótico e pró-inflamatório. A associação da SM com a doença cardiovascular aumenta a mortalidade geral em cerca de 1,5 vez.

A Federação Internacional de Diabetes (FID) publicou recentemente sua definição de SM em crianças e adolescentes. Esse painel recomenda os seguintes critérios: (1) para crianças de 6-10 anos de idade, a obesidade (definida como circunferência abdominal $\geq$ percentil 90), acompanhada por outras medidas, conforme indicadas pelo histórico familiar; (2) para a idade entre 10-16 anos, a obesidade (definida como circunferência abdominal $\geq$ percentil 90), seguida pelos critérios de adultos para triglicerídios, HDL-c, pressão arterial, e glicose; (3) Para jovens $\geq 16$ anos, o painel recomenda usar os mesmos critérios da FID para adultos. Essa definição se baseia em percentis de circunferência abdominal e é padrão para todas as faixas etárias previstas.

Usando os critérios do ATP III do NCEP e da OMS, estudos foram feitos, em meados da década de 2000, em diferentes países norte-americanos, com os seguintes resultados, que podem ser comparados: em escolas dos EUA, com 1.513 adolescentes, encontraram-se prevalências de SM de 4,2\% e 8,4\%, respectivamente; ${ }^{16}$ em 965 crianças e adolescentes mexicanos, encontraram-se os percentuais de $6,5 \%$ nas crianças e nos adolescentes, 4,5\%; ${ }^{17}$ no Canadá, avaliando 2.244 crianças e adolescentes, a prevalência de SM foi levemente superior (11.5\%). ${ }^{18}$

Apesar de ser bastante relevante o conhecimento sobre a prevalência da SM em outros países e em diferentes faixas etárias da população (crianças, adolescentes e adultos), sabe-se que a prevalência da SM nas diferentes populações depende muito dos critérios utilizados para sua definição e ainda não há estudos abrangentes, pelo menos não com dados significativos, sobre essa prevalência na população brasileira. Os estudos iniciais de prevalência da SM diferiram muito nos seus resultados, devido aos diversos critérios utilizados e porque selecionaram subgrupos específicos da população. Além disso, tem-se sugerido que cada população tenha essa definição validada de acordo com suas características étnicas locais. ${ }^{19}$

Entidades internacionais como FID (Federação Internacional de Diabetes), NHLBI (Instituto Nacional de Coração, Pulmões e Sangue, dos EUA), AHA (Associação Americana do Coração), WHF (Federação Mundial do Coração), IAS (Sociedade Internacional de Arteriosclerose) e IASO (Associação Internacional para Estudos da Obesidade) costumam divulgar documentos e declarações de consenso sobre o assunto ${ }^{8,9,20}$ para atualizar a comunidade científica sobre as mais recentes diretrizes pertinentes. O documento mais recente foi publicado em 2009 no periódico Circulation (v. 120, p. 640-1645) e deve ser utilizado como padrão-ouro nos trabalhos com SM. 
O documento mais recente ${ }^{9}$ é considerado uma diretriz internacional e representa o passo mais atual para a definição unificada da SM. Nele, é consenso que o indivíduo necessita apresentar, pelo menos, três dos cinco critérios clínicos definidos como componentes da SM para que seja possível firmar tal diagnóstico. A presença de qualquer um desses critérios não é obrigatória. Os pontos de corte de cada um dos critérios foram bem definidos, exceto para os valores da circunferência abdominal, que devem ser avaliados por cada país, respeitando sua etnia, nacionalidade e regionalização.

O papel da resistência à insulina como fator capaz de explicar todos os aspectos clínicos permanece ainda obscuro, tanto do ponto de vista da patogênese, como para um critério diagnóstico. As causas da SM permanecem vinculadas ao contexto social, econômico e cultural da população, sobretudo naquilo que se relaciona a atividade física e alimentação.

\section{Obesidade como fator de risco para SM}

A obesidade, definida como massa tecidual adiposa aumentada, geralmente se associa com inflamação crônica sistêmica, e confere altos riscos para câncer e desordens cardiovasculares e metabólicas. ${ }^{21}$ A obesidade é considerada uma epidemia mundial, e a Organização Mundial da Saúde ${ }^{22,23}$ a define como multifatorial, originando-se isoladamente ou na interção de fatores genéticos, sociais, culturais, nutricionais, metabólicos e/ou endócrinos. Segundo Conway \& René, ${ }^{24}$ a obesidade é uma doença complexa de etiologia multifacetada, com sua própria fisiopatologia, comorbidades e capacidades desabilitantes. No Brasil, as mudanças demográficas, socioeconômicas e epidemiológicas ao longo do tempo permitiram uma transição nos padrões nutricionais, com a diminuição progressiva da desnutrição e o aumento da obesidade. ${ }^{25}$

Ao longo da última década, tem havido crescente aumento da obesidade e comorbididade com a SM, embora pouco ainda se saiba realmente sobre a taxa de disfunção metabólica nesses casos. ${ }^{26}$ Vários fatores têm sido implicados como biomarcadores da SM, dentre os quais a insulina, o ácido úrico ${ }^{26} \mathrm{e}$ a proteína C-reativa (PCR), além de dois fatores diretamente ligados à obesidade por associação ao tecido adiposo, a adiponectina e a leptina. ${ }^{27,28}$

A obesidade tem sido associada com resistência à insulina ${ }^{29} \mathrm{e}$ é tida como o principal fator de risco para o desenvolvimento da pré-diabetes e do DMT2 ${ }^{30,31}$ e também a doença arterial coronariana (DAC). ${ }^{32}$ A maioria dos casos de SM ocorre em indivíduos com excesso de peso, o que, por si só, prejudica a sensibilidade à insulina, que diminui em até 40\% quando o indivíduo apresenta massa corporal maior que $35 \%$ a $40 \%$ da ideal..$^{33} \mathrm{O}$ excesso de gordura corporal leva ao acúmulo de lipídeos nos tecidos, notadamente no tecido adiposo, músculos, fígado e células $\beta$ pancreáticas, o que parece induzir as alterações bioquímicas ocorridas na SM. ${ }^{20}$ 


\section{Bases celulares e moleculares da função e disfunção adiposa}

O conhecimento dos eventos moleculares que regulam a diferenciação dos pré-adipócitos e de células-tronco mesenquimais em adipócitos (adipogênese) é importante para o entendimento da gênese da obesidade. A obesidade resulta do aumento no tamanho e no número de adipócitos, sendo que o balanço entre adipogênese e adiposidade ajuda a determinar o grau de obesidade do indivíduo. ${ }^{34} \mathrm{Na}$ obesidade, vários hormônios e citocinas parecem ter papel fundamental na manutenção do alto peso corpóreo.

Sabe-se que os adipócitos maduros secretam adipocinas ${ }^{i}$ ou adipocitocinas, cuja produção é bem maior na obesidade, o que contribui para o estabelecimento de resistência periférica à insulina. $\mathrm{Na} \mathrm{SM}$, o tecido adiposo parece funcionar como um órgão parácrino e endócrino, secretando várias adipocitocinas, tais como a adiponectina e a leptina, ${ }^{28}$ além das citocinas pró-inflamatórias clássicas (interleucina-6 ou IL-6, e o fator de necrose tumoral, TNF- $\alpha$ ), ${ }^{27}$ assim como lipocina, resistina, omentina, lipocalina e as proteínas adipocitárias de ligação a ácido graxo ou fatty acid binding proteins (A-FABP), dentre outras. Tais adipocitocinas são conhecidas por interagirem de forma bastante complexa.

A adiponectina e a leptina parecem estar diretamente associadas com obesidade e resistência à insulina. ${ }^{27,28}$ Deve-se mencionar o fato de que a adiponectina e a leptina parecem ter associações opostas com relação à SM e DAC..$^{35}$ Os adipócitos e macrófagos do tecido adiposo produzem Interleucina 6 (IL-6) em execesso, enquanto a expressão de fator necrosante tumoral alfa (TNF- $\alpha$ ) está aumentada na gordura visceral de obesos e se correlaciona positivamente com o grau de obesidade e com os níveis de insulina plasmática. ${ }^{36}$ IL-6 e TNF- $\alpha$ medeiam indiretamente a lipólise e aumentam a síntese hepática de ácidos graxos, de modo tal que aumentam os níveis séricos dos ácidos graxos e triglicérides. ${ }^{37} \mathrm{~A}$ cascata inflamatória disparada por essas citocinas é, por sua vez, ainda mais estimulada pela hiperinsulinemia. ${ }^{38}$ IL-6 e TNF- $\alpha$ também atuam diretamente no receptor de insulina para diminuir sua sinalização e, então, aumentar a resistência à insulina. ${ }^{39}$ Pode-se resumir explicando que o tecido adiposo visceral, aumentado na SM, perturba a secreção de adipocitocinas e leva a um estado inflamatório crônico de baixa intensidade, pela infiltração de macrófagos no tecido adiposo. ${ }^{43}$ Esse estado inflamatório se associa com a resistência à insulina e a aterosclerose. . $^{4,44,45}$

\footnotetext{
As citocinas pró-inflamatorias possuem efeitos metabólicos de longo alcance por todo o corpo, como alterações metabólicas das proteínas, gorduras e oligoelementos, além de alterações na síntese hepática das proteínas. ${ }^{40}$ As citocinas são definidas como proteínas solúveis sintetizadas por células imunes ou não, que medeiam a comunicação intracelular por transmitirem informações a células-alvo, via interações com receptores específicos. Muitas citocinas possuem atividades fisiológicas muito além daquelas originalmente descobertas. ${ }^{41}$ Atualmente, o termo mais aceito para descrever uma proteína que é secretada (e sintetizada) pelo tecido adiposo, sendo esta proteína uma citocina ou não, ${ }^{42}$ é adipocina.
} 
Para detalhar melhor, tem-se que a adiponectina possui propriedades antiaterogênicas, antidiabéticas e anti-inflamatórias, atenuando a resistência à insulina pelo aumento da sensibilidade insulínica no fígado. No tecido muscular, a adiponectina aumenta a utilização de glicose e a oxidação de ácidos graxos, além de aumentar a secreção de óxido nítrico (NO) endotelial e de inibir a adesão de monócitos e a proliferação de células da musculatura lisa da parede vascular. ${ }^{45,46,47}$

Quanto à leptina, ela também é uma citocina pró-inflamatória e parece ser importante na regulação perene da quantidade de gordura corporal. Ela é um hormônio peptídeo, produto do gene $o b$, que age como sinal aferente num loop de feedback negativo na regulação do tamanho da massa de tecido adiposo. ${ }^{28}$ A concentração de leptina, que também é um fator conhecido de saciedade, ${ }^{48}$ é proporcional ao número e tamanho dos adipócitos. ${ }^{49}$ Ela tem sido alvo recente de pesquisas para se esclarecer como a obesidade estimula a inflamação sistêmica através da ação e conexão de diversas citocinas, incluindo a própria leptina aumentada ${ }^{50} \mathrm{e}$ a adiponectina diminuída. ${ }^{45,46,47}$

Ainda sobre a leptina, recentes estudos demonstraram que a insulina e a leptina agem como reguladoras de um tipo de proteínas, chamadas aquagliceroporinas, que permitem o movimento de água e de outros solutos menores, em especial o glicerol, através das membranas celulares. O glicerol é um metabólito atuante no controle do acúmulo lipídico e na homeostase da glicose, importante como substrato para a gliconeogênese hepática, para secreção pancreática de insulina e para a produção cardíaca de ATP. As aquagliceroporinas (AQP3, AQP7, AQP9 e AQP10) compreendem uma subfamília das aquaporinas. ${ }^{50,51}$ Através de uma via metabólica conhecida pela sigla PI3K/Akt/mTOR (Phosphatidylinositol 3-Kinase/Akt/mammalian target of rapamycin), e considerando que o tecido adiposo constitui uma fonte primordial de glicerol via AQP $7,{ }^{49,53}$ relatos mais recentes têm evidenciado que as aquagliceroporinas (em particular AQP3 e AQP7) parecem facilitar o efluxo de glicerol a partir do tecido adiposo, ao mesmo tempo em que podem reduzir o influxo de glicerol para o interior dos hepatócitos via AQP9, prevenindo o excesso de lipídios e o subsequente agravamento da hiperglicemia na obesidade humana. ${ }^{51,52}$ Conforme ilustra a figura 1, extraída do artigo de Rodríguez et al., ${ }^{51}$ pode-se constatar que funções tão versáteis e relevantes das aquagliceroporinas deixam transparecer os papéis inesperados e emergentes desses canais de glicerol.

Outras adipocinas recém-reportadas ${ }^{54,55}$ são: vaspina, visfatina, apelina, omentina, fractalquina, proteína estimulante de acilação (acylation stimulating protein, ASP) e proteína de ligação ao retinol (retinol-binding protein 4, RBP4). O papel, ora benéfico ora deletério, dessas adipocinas na obesidade e aterosclerose, tem sido bastante estudado. A vaspina (acrônimo para visceral adipose tissue-derived serine protease inhibitor) foi identificada como membro da família protéica de inibidores serino-protease, podendo ter efeitos antiaterogênicos através de suas propriedades sensibilizadoras de insulina. 
Do mesmo modo, a visfatina também possui essas propriedades, mas ela parece desestabilizar placas ateromatosas, o que vem a ser um efeito deletério. A apelina, via inibição do consumo alimentar e aumento na atividade física e temperatura corporal, pode promover perda de peso, resultando em efeito benéfico antiaterogênico, que se associa a outros efeitos positivos na vasodilatação e pressão sanguínea. Considerando os níveis elevados de RBP4 em indivíduos ateroscleróticos, essa proteína pode constituir um bom biomarcador. Outrossim, a ASP, geralmente aumentada na obesidade e SM, pode contribuir no armazenamento eficiente de lipídios, podendo o decréscimo ou bloqueio dessa proteína fornecer um alvo antiobesidade em potencial.

Quanto à omentina-1, a isoforma mais comum, foi identificada como uma nova adipocina

predominantemente secretada pelas células vasculares do estroma visceral, embora não seja produzida exatamente pelos adipócitos. ${ }^{55}$ Experimentos in vitro revelaram que o tratamento com omentina-1 recombinante acentua a captação de glicose mediada por insulina em adipócitos humanos subcutâneos e do omento, ao mesmo tempo em que aumenta a fosforilação de Akt/PKB. Já estudos com pacientes humanos portadores de T1DM demonstraram o decréscimo da concentração plasmática de omentina-1 não afetada por ingestão de glicose. Ademais, a concentração plasmática e a expressão gênica de omentina no tecido adiposo visceral parecem decrescer na obesidade.

\section{Tecido adiposo visceral e adipocinas}

Inúmeros estudos clínicos e experimentais, especialmente na última década, enfocaram o tecido adiposo visceral e a obesidade abdominal. ${ }^{45,46}$ A gordura abdominal é composta por gordura subcutânea e visceral, a qual, dependendo do tipo predominante de gordura, é classificada como obesidade abdominal subcutânea ou visceral, sendo esta a de maior risco para a saúde. Conforme revisto, ${ }^{56}$ o tecido adiposo visceral (TAV) ou omental é o mais ativo, ou seja, mais sensível à lipólise, via catecolaminas e $\beta$-adrenorreceptores, e mais resistente à ação da insulina, liberando maior concentração de ácidos graxos livres (AGL), diretamente na veia porta. Além disso, o TAV secreta maiores concentrações de adipocinas ligadas a processos pró-inflamatórios como resistina, angiotensina I, inibidor plasminogênio ativado 1 (PAI-1), RCP, IL-6, seguido do tecido adiposo subcutâneo abdominal (TASA) e do tecido adiposo subcutâneo glúteo-femural (TASG).

Em relação aos depósitos específicos de gordura corporal, expressões duas a três vezes maiores de resistina, uma das adipocinas já citadas, são encontradas no tecido adiposo visceral, seguido de subcutâneo abdominal e subcutâneo glúteo-femural, podendo o aumento da expressão de resistina ser um importante elo entre obesidade abdominal e DMT2. Além disso, sua expressão é três vezes maior nos pré-adipócitos quando comparada aos adipócitos maduros, funcionando também como potencial reguladora da adipogênese. ${ }^{57}$ 
A circunferência abdominal é uma medida recomendada por representar um índice antropométrico representativo da gordura intra-abdominal e de aferição simples e reprodutível..$^{58}$ A aferição desse índice se faz pela medida no meio da distância entre a crista ilíaca e o rebordo costal inferior (SBC, 2005). Para circunferência de cintura abdominal entre 80-88 cm e 94-102 $\mathrm{cm}$, respectivamente para mulheres e homens, recomenda-se monitorização mais frequente dos fatores de risco para doenças coronarianas. ${ }^{58}$

Sabe-se que as adipocinas podem atuar como um elo entre adiposidade visceral e aterosclerose. ${ }^{34}$ Nos depósitos viscerais de gordura, há turnover mais acelerado que em outras regiões, elevando as concentrações de PAI-1, citocinas inflamatórias e ácidos graxos não esterificados, segundo DECS (NEFA) no sistema porta. A maior liberação de NEFA do tecido adiposo estimula a gliconeogênese, inibe a depuração hepática de insulina e provoca acúmulo de triglicérides no fígado e músculo, resultando em hiperglicemia e consequente hiperinsulinemia. Assim, o acúmulo de gordura no músculo leva à resistência insulínica, enquanto no fígado, promove a dislipidemia aterogênica. ${ }^{33,59}$

\section{Considerações finais}

A SM tem-se mostrado com alta prevalência mundial e ocorrendo nos grupos de sendentários e obesos. O conhecimento das bases moleculares que regulam a inflamação, obesidade e suas comorbidades, pode proporcionar novas abordagens terapeuticas no tratamento ou prevenção destas patologias.

\section{Referências}

1. Kylin E. Studien uber das Hypertonie-Hyperglyka "mie-Hyperurika" miesyndrom. Zentralbl Inn Med. 1923; 44: 105-27.

2. Steinberger J. et al. Progress and challenges in metabolic syndrome in children and adolescents: a scientific statement from the American Heart Association Atherosclerosis, Hypertension, and Obesity in the Young Committee of the Council on Cardiovascular Disease in the Young; Council on Cardiovascular Nursing; and Council on Nutrition, Physical Activity, and Metabolism. Circulation. 2009; 119(4): 628-47.

3. Kaplan NM. The deadly quartet: upper-body obesity, glucose intolerance, hypertriglyceridemia and hypertension. Arch Intern Med. 1989; 149: 1514-20.

4. Defronzo RA, Ferrannini E. Insulin resistance: a multifaceted syndrome responsible for NIDDM, obesity, hypertension, dyslipidemia, and atherosclerotic cardiovascular disease. Diabetes Care. 1991; 14: 173-94.

5. Björntorp P. Abdominal obesity and the metabolic syndrome. Ann Méd. 1991; 24: 465-8. 
6. Reaven GM. Pathophysiology of insulin resistance in human disease. Physiol Rev. 1995; 75: 473-86.

7. Després JP, Lemieux I. Abdominal obesity and metabolic syndrome. Nature. 2006; 444: 881-7.

8. Grundy SM et al. Diagnosis and management of the metabolic syndrome: an American Heart Association/National Heart, Lung, and Blood Institute Scientific Statement [published corrections appear in Circulation. 2005;112:e297 and 2005;112:e298]. Circulation. 2005; 112: 2735-52.

9. Grundy SM et al. Definition of metabolic syndrome: report of the national heart, lung, and blood institute/ American Heart Association Conference on Scientific issues related to definition. Circulation. 2009; 109(3): 433-8.

10. Sociedade Brasileira de Cardiologia. I Diretriz de Prevenção da Aterosclerose na Infância e na Adolescência. Arq Bras Cardiol. 2005; 85 supl. 6, S4-36.

11. Bonora E, Kiechl S, Willeit J, Oberhollenzer F, Egger G, Bonadonna CR, et al. Metabolic Syndrome: epidemiology and more extensive phenotypic description. Cross-sectional data from the Bruneck Study. Int J Obes. 2003; 27: 1283-9.

12. World Health Organization. Department Of Noncommunicable Disease Surveillance. Report of a WHO Consultation: Definition of Metabolic Syndrome in Definition, Diagnosis, and Classification of Diabetes Mellitus and Its Complications: Report of a WHO Consultation, Part 1: Diagnosis and Classification of Diabetes Mellitus. Geneva: World Health Organization; 1999.

13. World Health Organization. Diet, nutrition and the prevention of chronic diseases. Geneva; 2003. $149 \mathrm{p}$.

14. Alberti KGMM, et al. Harmonizing the Metabolic Syndrome. A Joint Interim Statement of the International Diabetes Federation Task Force on Epidemiology and Prevention; National Heart, Lung, and Blood Institute; American Heart Association; World Heart Federation; International Atherosclerosis Society; and International Association for the Study of Obesity. Circulation. 2009; 120: $1640-5$.

15. Lorenzo C. The metabolic syndrome as predictor of type 2 diabetes: the San Antonio heart study. Diabetes Care. 2003; 26(11): 3153-9.

16. Goodman E, Daniels SR, Morrison JA, Huang B, Dolan LM. Contrasting prevalence of and demographic disparities in the World Health Organization and National Cholesterol Education Program Adult Treatment Panel III definitions of metabolic syndrome among adolescents. J Pediatr. 2004; 145: 445-51.

17. Rodríguez-Morán M, Salazar-Vázquez B, Violante R, Guerrero-Romero F. Metabolic Syndrome among children and adolescents aged 10-18 years. Diabetes Care. 2004; 27: 2516-7.

18. Lambert M, Paradis G, O’Jloughlin J, Delvin EE, Hanley JA, Levy E. Insulin resistance syndrome in a representative sample of children and adolescents from Quebec, Canada. Int J Obes Relat Metab Disord. 2004; 28:833-41.

19. Rosenbaum P, Gimeno SG, Sanudo A, Franco LJ, Ferreira SR. Analysis of criteria for metabolic syndrome in a population-based study of Japanese-Brazilians. Diabetes Obes Metab. 2005; 7:352-9.

20. Grundy SM, et al. Implications of recent clinical trials for the National Cholesterol Education Program Adult Treatment Panel III guidelines. Circulation. 2004; 110(2): 227-39. 
21. Olefsky JM, Glass CK. Macrophages, Inflammation, and Insulin Resistance. Annu Rev Physiol. 2010 mar.; 72: 219-46.

22. World Health Organization. Obesity - preventingand managing the global epidemic. 2000.

23. Word Health Organization. Word Health Report. Reducing risks, promoting healthy life. Geneva: WHO; 2002.

24. Conway B, Rene A. Obesity as a disease: no lightweight matter. Obes Rev. 2004; 5: 145-51.

25. Monteiro CA. Velhos e novos males da saúde no Brasil: a evolução do pais e suas doenças. São Paulo: HUCITEC NUPENS/USP; 1995. v.1, p. 421-30.

26. Abdullah AR, Hasan HA, Raigangar VL. Analysis of the relationship of leptin, high-sensitivity C-reactive protein, adiponectin, insulin, and uric acid to metabolic syndrome in lean, overweight, and obese young females. Metab Synndr Relat Disord Metab. 2009 fev.; 7(1): 17-22.

27. Pittas AG, Joseph NA, Greenberg AS. Adipocytokines and Insulin. J Clin Endocrinol Metab. 2004 fev.; 89(2): 447-52.

28. Sahu A. Minireview: A hypothalamic role in energy balance with special emphasis on leptin. Endocrinology. 2004; 145: 2613-20.

29. Defronzo RA, Bonadonna RC, Ferrannini E. Pathogenesis of NIDDM: a balanced overview. Diabetes Care. 1992; 15:318-68.

30. Hu FB, Manson JE, Stampfer MJ, Colditz G, Liu S, Solomon CG, et al. Diet, lifestyle and the risk of type 2 diabetes mellitus in women. N Engl J Med. 2001; 345: 790-7.

31. Gómez-Ambrosi J, Silva C, Galofré JC, Escalada J, Santos S, Gil MJ, Valentí V, et al. Body Adiposity and Type 2 Diabetes: Increased Risk With a High Body Fat Percentage Even Having a Normal BMI. Obesity 2011 Mar. Silver Spring; 10.

32. Rexrode KM, Manson JE, Hennekens CH. Obesity and cardiovascular disease. Curr Opin Cardiol. 1996; 11: 490-5.

33. Santos CR, Portella ES, Avila SS, Soares EA. Identificação da síndrome metabólica em diabéticos tipo dois atendidos em Hospital Universitário do Rio de Janeiro. Rev Soc Cardiol Estado de São Paulo. 2003; 13(2 Edição Especial): 98.

34. Queiroz JCF, Alonso-Vale MIC, Curi R, Lima FB. Controle da adipogênese por ácidos graxos. Arq Bras Endocrinol Metab [online]. 2009 [cited 2012-02-24]; 53(5): 582-94.

35. Hall JI, et al. Leptin/adiponectin ratio in patients with coronary heart disease: comparing subjects with and without metabolic syndrome. Ann Clin Biochem. 2011 Apr; 18. [Epub ahead of print]

36. Le Roith D, Zick Y. Recent advances in our understanding of insulin action and insulin resistance. Diabetes Care. 2011; 24: 588-97.

37. Khovidhunkit W, et al. Infection and inflammation-induced proatherogenic changes of lipoproteins. J Infect Dis. 2000; 181(suppl 3): S462-72. 
38. Hotamisligil GS, Shargill NS, Spiegelman BM. Adipose expression of tumor necrosis factor-alpha: direct role in obesity-linked insulin resistance. Science. 1993 [Acesso em 18 jan 2012]; 259: 87-91. Disponível em: http://www.ncbi.nlm.nih.gov/entrez/query.fcgi?db=pubmed\&.

39. Marette A. Mediators of cytokine-induced insulin resistance in obesity and other inflammatory settings. Curr Opin Clin Nutr Metab Care. 2002; 5:377-83. 2002.

40. Bistrian BR, GrimbleRF. Nutrition and immune and inflammatory systems. In: Gibney MJ, Elia M, Ljungqvist O, Dowsett J [organizadores]. Clinical Nutrition. Oxford: Blackwell Publishing; 2005. p. 247-67.

41. Fruhbeck G, Gómez-Ambrosi J, Muruzábal FJ, Burrell MA. The adipocyte: a model for integration of endocrine and metabolic signaling in energy metabolism regulation. Endocrinology and Metabolism. 280(6): E827-47.

42. Trayhurn P, Wood IS. Adipokines: inflammation and the pleiotropic role of white adipose tissue. $\mathrm{Br}$ J Nutr. 2004 Sep; 92(3): 347-55.

43. Galic S, Oakhill JS, Steinberg GR. Adipose tissue as an endocrine organ. Mol Cell Endocrinol. 2010; 316:129-39.

44. Maury E, Brichard SM. Adipokine dysregulation, adipose tissue inflammation and metabolic syndrome. Mol Cell Endocrinol. 2010 jan.; 314(1): 1-16.

45. Gormez S, et al. Adipose Tissue Gene Expression of Adiponectin, Tumor Necrosis Factor- $\alpha$ and Leptin in Metabolic Syndrome Patients with Coronary Artery Disease. Intern Med. 2011;50: 805-10.

46. Chandran $\mathrm{M}$ et al. Adiponectin: more than just another fat cell hormone? Diabetes Care, v. 26, p. 2442-2450, 2003.

47. Diez J.J., Iglesias P. The role of the novel adipocyte-derived hormone adiponectin in human disease. Eur J Endocrinol, v.148, p.293-300. 2003.

48. Fruhbeck G. A heliocentric view of leptin. Proc Nutr Soc. 2001; 60: 301-18.

49. Fruhbeck $G$, et al. Body adiposity and type 2 diabetes: increased risk with a high body fat percentage even having a normal BMI. Obesity. 2011 Mar. (Silver Spring); 10.

50. Heber D, Carpenter CL. Addictive genes and the relationship to obesity and inflammatio molecular neurobiology. 2011 apr.; 44(2): 160-5.

51. Rodríguez A, Catalán V, Gómez-Ambrosi J, García-NavarroS, Rotellar F, Valentí V, et al. Insulin- and Leptin-Mediated Control of Aquaglyceroporins in Human Adipocytes and Hepatocytes Is Mediated via the PI3K/Akt/mTOR Signaling Cascade. J Clin Endocrinol Metab. 2011 Apr; 96(4): E586-97. Epub 2011 Feb 2.

52. Rodríguez A, Catalán V, Gómez-Ambrosi J, Fruhbeck G. Aquaglyceroporins serve as metabolic gateways in adiposity and insulin resistance control. Cell Cycle. 2011 May 15;10(10).

53. Kishisa K, et al. Aquaporin adipose, a purative glycerol channel in adipocytes. J Biol Chem. 2000; 275:20896-902. 
54. Gauvreau D, Villeneuve N, Deshaies Y. Novel adipokines: links between obesity and atherosclerosis. Cianflone KAnn Endocrinol (Paris). 2011 Jun; 72(3): 224-31.

55. Auguet T, Quintero Y, Riesco D, Morancho B, Terra X, Crescenti A, et al. New adipokines vaspin and omentin. Circulating levels and gene expression in adipose tissue from morbidly obese women. BMC Med Genet. 2011 apr.; 12: 1-8.

56. Hermsdorff HHM, Monteiro JBR. Gordura visceral subcutânea ou intramuscular : onde está o problema? Arq Bras Endocrinol Metab. 2004; 48(6): 803-11.

57. Mattison R, Jensen M. The adipocyte as an endocrine cell.Current opinion in endocrinology, diabetes and obesity. 2003 Out.; 10(5): 317-21.

58. Janssen I, Katzmarzyk PT, Ross R. Body mass index, waist circumference, andhealth risk. Arch Intern Med. 2002; 162: 2074-9.

59. Barbalho SM, McLellan KCP, Lerario AC. A síndrome metabólica e sua relação com a resistência à insulina, disfunção endotelial e aterogênese. Nutrire Rev Soc Bras Aliment Nutr. 2007 abr.; 32(1): 89-100.

Recebido: 09/8/2012

Revisado: $10 / 11 / 2013$

Aprovado: $14 / 1 / 2013$ 